\title{
Adaptive Iterative Learning Control of Non-uniform Trajectory Tracking for Strict Feedback Nonlinear Time-varying Systems
}

\author{
Chun-Li Zhang Jun-Min Li \\ Department of Applied Mathematics, Xidian University, Xi' an 710071, China
}

\begin{abstract}
In this paper, an iterative learning control strategy is presented for a class of nonlinear time-varying systems, the timevarying parameters are expanded into Fourier series with bounded remainder term. The backstepping design technique is used to deal with system dynamics with non-global Lipschitz nonlinearities and the approach proposed in this paper solves the non-uniform trajectory tracking problem. Based on the Lyapunov-like synthesis, the proposed method shows that all signals in the closed-loop system remain bounded over a pre-specified time interval $[0, T]$. And perfect non-uniform trajectory tracking of the system output is completed. A typical series is introduced in order to deal with the unknown bound of remainder term. Finally, a simulation example shows the feasibility and effectiveness of the approach.
\end{abstract}

Keywords: Iterative learning control, time-varying systems, Lyapunov-like, non-uniform trajectory tracking, Fourier series expansion, backstepping.

\section{Introduction}

Iterative learning control (ILC) or adaptive iterative learning control (AILC) has become one of the most effective control strategies in dealing with repeated tracking control of nonlinear systems. And the additional requirement of the repetitive mode is that a specified output trajectory on a finite interval is followed with a high precision (or so called exactly tracking). Examples of such systems include robotic manipulators required to repeat a given task with high precision, chemical batch processes, vehicles and man-machine systems. The ILC system improves the control performances by self-tuning the leaning gains in the traditional D-type, P-type, PD-type or PID-type ILC for linear or affine nonlinear dynamic systems with nonlinearities satisfying the global Lipschitz continuous condition ${ }^{[1,2]}$.

In the existing literature, the tracking trajectory must be uniform, for a complex tracking trajectory problem (such as varying trajectory along iterative direction), there is no well-posed method to consider it. Actually, the non-uniform trajectory can be considered as an uncertain time-varying parameter along both iterative direction and time domain, therefore, it becomes a challenging problem to study an ILC strategy for uncertain time-varying parametric systems. In [3], D-type, PD-type and PID-type learning algorithms were presented to solve the problem of slow varying trajectory along iterative direction. In [4], an adaptive iterative learning control method via Lyapunov technique was proposed for the system with unknown constant parameter uncertainty, which can be used to track the similar variant trajectory or unseen trajectories. In [5], a new adaptive iterative learning control strategy was presented on the basis

Regular paper

Manuscript received July 30, 2012; accepted February 14, 2014

This work was supported by National Natural Science Foundation of China (No. 60974139) and Fundamental Research Funds for the Central Universities (No. 72103676). of neural networks, which has the capability of generalization both for tracking trajectory and for system structure. However, there still have some restricts on the trajectory. In [6], for the first-order hybrid parametric system, a new iterative learning control law consisting of a feedback term and a learning term was proposed, which can perform different tracking control tasks. Recently, a novel adaptive iterative learning control approach was proposed for a class of hybrid parametric nonlinear systems by means of backstepping method in [7]. Similarly, a novel adaptive iterative learning control approach was proposed for a class of hybrid parametric nonlinear time-delay systems ${ }^{[8]}$. The approach consists of a differential-deference type updating law and a learning control law for handling the non-uniform trajectory tracking problem. It avoids the restrictions on the tracking trajectory in the traditional ILC.

In many industrial applications, the system parameters are completely unknown or partially unknown. When these parameters are unknown time-varying ones, the controller design problem of the uncertain nonlinear system becomes a challenging topic ${ }^{[9-11]}$. When the period of uncertain parameters of the system is known in advance, by the pointwise integral mechanism, a new adaptive control approach characterized by periodic parameter adaptation was proposed, which complements periodic parameter adaptation control of the first-order uncertain system with mixed linear parameters, such that the tracking error converges to zero asymptotically in the $L_{T}^{2}$-norm sense ${ }^{[12]}$. In [13], a new approach of designing a repetitive learning controller for a class of unmatched nonlinear systems with both completely unknown virtual control coefficients and unknown time-varying parameters was proposed, which by incorporating a Nussbaum-type function and backstepping technique, can guarantee uniform ultimate boundedness of the states. In [14], combining the backstepping approach with the pointwise integral mechanism, a novel adaptive repeti- 
tive learning control for high-order nonlinear systems with time-varying and time-invariant parameters was proposed. An iterative learning controller was presented for a class of strict-feedback nonlinear systems with time-varying uncertainties in [15]. The learning controller is designed based on the Lyapunov-like synthesis, which can handle system dynamics with non-global Lipschitz nonlinearities. The theoretical analysis shows that all signals in the closed-loop system remain bounded over a pre-specified time interval $[0, T]$. And complete tracking of the system output is achieved. But the control objective of the article was uniform and the bound of remain term was known.

Motivated by the above discussion, we propose an iterative learning controller for a class of nonlinear time-varying systems to solve the non-uniform trajectory tracking problem. The learning controller is designed based on the Lyapunov-like synthesis, which can handle system dynamics with non-global Lipschitz nonlinearities. For the controller design, the time-varying parameters are expanded into Fourier series with bounded remainder term, whose bound is unknown. The theoretical analysis shows that all signals in the closed-loop system remain bounded, and perfect non-uniform trajectory tracking of the system output is completed. Finally, we give a simulation example to show the feasibility and effectiveness of the approach.

\section{System description}

Consider a class of high-order nonlinear time-varying systems

$$
\begin{aligned}
& \dot{x}_{i}=x_{i+1}+\theta^{\mathrm{T}}(t) \varphi_{i}\left(\bar{x}_{i}\right) \\
& \dot{x}_{n}=u(t)+\theta^{\mathrm{T}}(t) \varphi_{n}\left(\bar{x}_{n}\right) \\
& y=x_{1}
\end{aligned}
$$

where $\bar{x}_{n}=\left[x_{1}, \cdots, x_{n}\right]^{\mathrm{T}} \in \mathbf{R}^{n}$ is the state vectors of the system, which is assumed to be available for measurement, $\bar{x}_{i}=\left[x_{1}, \cdots, x_{i}\right]^{\mathrm{T}}, u \in \mathbf{R}$ and $y \in \mathbf{R}$ correspond to system input and output, respectively. Denote $x=\bar{x}_{n} . x\left(t_{0}\right)=x_{0}$ represents initial conditions of the system, $\theta(t) \in \mathbf{R}^{p}$ is an unknown continuous time varying function vector, $\varphi_{i}\left(\bar{x}_{i}\right)$, $i=1, \cdots, n$ are all known smooth function, and $\varphi_{i}(0)=0$, $i=1, \cdots, n$.

Now, we suppose that the system time-varying parameter vector $\theta(t)$ is a fully unknown continuous periodic function vector with a known period $T$. Each component $\theta_{i}(t), i=$ $1, \cdots, p$ of the continuous and periodic function vector $\theta(t)$ can be expressed by a linearly parameterized Fourier series expansion as

$$
\theta_{i}(t)=\phi_{i}^{\mathrm{T}}(t) \eta_{i}+\delta_{i}(t),\left|\delta_{i}(t)\right| \leqslant \bar{\delta}_{i}
$$

where $\eta_{i}=\left[\eta_{i 1}, \eta_{i 2}, \cdots, \eta_{i q}\right]^{\mathrm{T}} \in \mathbf{R}^{q}$ is a constant vector consisting of the first $q$ coefficients of the Fourier series expansion of $\theta_{i}(t)$ ( $q$ is an odd integer). $\delta_{i}(t)$ is the truncation error with the minimum upper bound $\bar{\delta}_{i}>0$, which can be arbitrarily decreased by increasing $q$. And $\phi_{i}(t)=$ $\left[\phi_{i 1}(t), \cdots, \phi_{i q}(t)\right]^{\mathrm{T}}$ with $\phi_{i 1}(t)=1, \phi_{i 2 j}(t)=\sin \left(\frac{2 \pi j t}{T}\right)$ and $\phi_{i 2 j+1}(t)=\cos \left(\frac{2 \pi j t}{T}\right), j=1, \cdots, \frac{(q-1)}{2}$, whose derivatives up to $n$-order are smooth and bounded.

Let $\phi(t)=\operatorname{diag}\left\{\phi_{1}(t), \phi_{2}(t), \cdots, \phi_{n}(t)\right\}, \eta=\left[\eta_{1}^{\mathrm{T}}, \eta_{2}^{\mathrm{T}}, \cdots\right.$ ,$\left.\eta_{n}^{\mathrm{T}}\right]^{\mathrm{T}}, \delta(t)=\left[\delta_{1}(t), \delta_{2}(t), \cdots, \delta_{n}(t)\right]^{\mathrm{T}}$. Then the parameter vector $\theta(t)$ can be rewritten as

$$
\theta(t)=\phi^{\mathrm{T}}(t) \eta+\delta(t)
$$

From (2), we have $\|\delta(t)\| \leqslant s$. We assume that the upper bound $s$ is an unknown parameter. Then after substituting (3) into system (1), system (1) becomes

$$
\begin{aligned}
& \dot{x}_{i}=x_{i+1}+\eta^{\mathrm{T}} \phi(t) \varphi_{i}\left(\bar{x}_{i}\right)+\delta^{\mathrm{T}}(t) \varphi_{i}\left(\bar{x}_{i}\right) \\
& \dot{x}_{n}=u(t)+\eta^{\mathrm{T}} \phi(t) \varphi_{n}\left(\bar{x}_{n}\right)+\delta^{\mathrm{T}}(t) \varphi_{n}\left(\bar{x}_{n}\right) \\
& y=x_{1} .
\end{aligned}
$$

Our objective is to design an adaptive iterative learning control law $u_{k}(t)$ on $[0, T]$, such that the tracking error $e_{k}(t)=y_{k}(t)-y_{r, k}(t)$ converges to zero completely when $k \rightarrow \infty$, and all signals of the closed-loop system are kept bounded, where $k$ denotes the iteration index, $y_{r, k}(t)$ represents the $k$-th sufficiently smooth iterative target trajectory.

In order to design the controller, a definition of convergent series sequence and its lemma are given as follows.

Definition $\mathbf{1}^{[15]}$. A convergent series sequence $\left\{\Delta_{k}\right\}$ is defined as

$$
\Delta_{k}=\frac{a}{k^{l}}
$$

where $k=1,2, \cdots ; a$ and $l$ are designed constant parameters, and $a>0 \in \mathbf{R}, l \geqslant 2 \in \mathbf{N}$.

Lemma $\mathbf{1}^{[15]}$. For given sequence $\left\{\frac{1}{k^{l}}\right\}$, where $k=$ $1,2, \cdots$, and the positive integer $l \geqslant 2$, the following inequality holds:

$$
\lim _{k \rightarrow \infty} \sum_{i=1}^{k} \frac{1}{i^{l}} \leqslant 2 .
$$

\section{Adaptive iterative learning controller design}

In the design process of the controller, we introduce the convergent series sequence of Definition 1 to eliminate the effect on system performance of the redundant item after expanding the time-varying parameter by using Fourier series. We give the design process of the controller for highorder strict feedback nonlinear time-varying systems as follows.

\subsection{Design of the controller}

Step 1. Let $\omega_{1, k}=\varphi_{1, k}$. There exists a smooth function $\bar{\varphi}_{1, k}\left(x_{1, k}\right)>0$ such that $\left|\delta^{\mathrm{T}}(t) \varphi_{1, k}\left(x_{1, k}\right)\right| \leqslant$ $\left\|\delta^{\mathrm{T}}(t)\right\| \bar{\varphi}_{1, k}\left(x_{1, k}\right) \leqslant s \bar{\varphi}_{1, k}\left(x_{1, k}\right)$. Denote $S=s^{2}, z_{1, k}=$ $x_{1, k}-y_{r, k}, z_{2, k}=x_{2, k}-\alpha_{1, k}-\dot{y}_{r, k}$, where $\alpha_{1, k}$ is the virtual controller. The time derivative of $z_{1, k}$ along systems (4) is given as

$$
\dot{z}_{1, k}=z_{2, k}+\alpha_{1, k}+\eta^{\mathrm{T}} \phi(t) \omega_{1, k}+\delta^{\mathrm{T}}(t) \omega_{1, k} .
$$

For any real number $a>0$ and positive integer $l \geqslant 2$, let $\Delta_{k}=\frac{a}{k^{l}}$, and denote $\tau_{1, k}=\Gamma_{1} \phi(t) \omega_{1, k} z_{1, k}, \nu_{1, k}=$ $\Gamma_{2} \frac{1}{\Delta_{k}} \bar{\varphi}_{1, k}^{2} z_{1, k}^{2}$.

Take virtual control as $\alpha_{1, k}=-c_{1} z_{1, k}-\hat{\eta}_{k}^{\mathrm{T}} \phi(t) \omega_{1, k}-$ $\hat{S}_{k} \frac{1}{\Delta_{k}} \bar{\varphi}_{1, k}^{2} z_{1, k}$, where $c_{1}$ is a positive constant. It is substi- 
tuted into (7), and we have

$$
\begin{aligned}
\dot{z}_{1, k}= & z_{2, k}-c_{1} z_{1, k}+\tilde{\eta}_{k}^{\mathrm{T}} \phi(t) \omega_{1, k}- \\
& \hat{S}_{k} \frac{1}{\Delta_{k}} \bar{\varphi}_{1, k}^{2} z_{1, k}+\delta^{\mathrm{T}}(t) \omega_{1, k}
\end{aligned}
$$

where $\hat{\eta}_{k}$ is the estimation of parameter $\eta$, and $\tilde{\eta}_{k}=\eta-\hat{\eta}_{k}$ is parameter estimation error. Take the following nonnegative function:

$$
V_{1, k}\left(z_{k}, \hat{\eta}_{k}, \hat{S}_{k}\right)=\frac{1}{2} z_{1, k}^{2}+\frac{1}{2} \tilde{\eta}_{k}^{\mathrm{T}} \Gamma_{1}^{-1} \tilde{\eta}_{k}+\frac{1}{2} \Gamma_{2}^{-1} \tilde{S}_{k}^{2}
$$

where $\Gamma_{1}$ and $\Gamma_{2}$ are symmetric positive definite matrices. $\hat{S}_{k}$ is the estimation of parameter $S, \tilde{S}_{k}=S-\hat{S}_{k}$ is the parameter estimation error. The time derivative of $V_{1, k}$ along systems (8) is given as

$$
\begin{aligned}
\dot{V}_{1, k}= & z_{1, k} z_{2, k}-c_{1} z_{1, k}^{2}+\tilde{\eta}_{k}^{\mathrm{T}} \Gamma_{1}^{-1}\left(\tau_{1, k}-\dot{\hat{\eta}}_{k}\right)- \\
& \hat{S}_{k} \frac{1}{\Delta_{k}} \bar{\varphi}_{1, k}^{2} z_{1, k}^{2}+\delta^{\mathrm{T}}(t) \omega_{1, k} z_{1, k}-\Gamma_{2}^{-1} \tilde{S}_{k} \dot{\hat{S}}_{k} \leqslant \\
& z_{1, k} z_{2, k}-c_{1} z_{1, k}^{2}+\tilde{\eta}_{k}^{\mathrm{T}} \Gamma_{1}^{-1}\left(\tau_{1, k}-\dot{\hat{\eta}}_{k}\right)- \\
& \hat{S}_{k} \frac{1}{\Delta_{k}} \bar{\varphi}_{1, k}^{2} z_{1, k}^{2}+s \bar{\varphi}_{1, k}\left|z_{1, k}\right|-\Gamma_{2}^{-1} \tilde{S}_{k} \dot{\hat{S}}_{k} \leqslant \\
& z_{1, k} z_{2, k}-c_{1} z_{1, k}^{2}+\tilde{\eta}_{k}^{\mathrm{T}} \Gamma_{1}^{-1}\left(\tau_{1, k}-\dot{\hat{\eta}}_{k}\right)- \\
& \hat{S}_{k} \frac{1}{\Delta_{k}} \bar{\varphi}_{1, k}^{2} z_{1, k}^{2}+\frac{1}{\Delta_{k}} s^{2} \bar{\varphi}_{1, k}^{2} z_{1, k}^{2}+ \\
& \frac{1}{4} \Delta_{k}-\Gamma_{2}^{-1} \tilde{S}_{k} \dot{\hat{S}}_{k}= \\
& z_{1, k} z_{2, k}-c_{1} z_{1, k}^{2}+\tilde{\eta}_{k}^{\mathrm{T}} \Gamma_{1}^{-1}\left(\tau_{1, k}-\dot{\hat{\eta}}_{k}\right)- \\
& \hat{S}_{k} \frac{1}{\Delta_{k}} \bar{\varphi}_{1, k}^{2} z_{1, k}^{2}+\frac{1}{\Delta_{k}} S \bar{\varphi}_{1, k}^{2} z_{1, k}^{2}+ \\
& \frac{1}{4} \Delta_{k}-\Gamma_{2}^{-1} \tilde{S}_{k} \dot{\hat{S}}_{k}= \\
& z_{1, k} z_{2, k}-c_{1} z_{1, k}^{2}+\tilde{\eta}_{k}^{\mathrm{T}} \Gamma_{1}^{-1}\left(\tau_{1, k}-\dot{\hat{\eta}}_{k}\right)+ \\
& \frac{1}{4} \Delta_{k}+\frac{1}{\Delta_{k}} \tilde{S}_{k} \bar{\varphi}_{1, k}^{2} z_{1, k}^{2}-\Gamma_{2}^{-1} \tilde{S}_{k} \dot{\hat{S}}_{k}= \\
& z_{1, k} z_{2, k}-c_{1} z_{1, k}^{2}+\tilde{\eta}_{k}^{\mathrm{T}} \Gamma_{1}^{-1}\left(\tau_{1, k}-\dot{\hat{\eta}}_{k}\right)+ \\
& \tilde{S}_{k} \Gamma_{2}^{-1}\left(\nu_{1, k}-\dot{\hat{S}}_{k}\right)+\frac{1}{4} \Delta_{k} .
\end{aligned}
$$

The following inequality is used in the previous equation. For any $r>0, m n \leqslant \frac{1}{r} m^{2}+\frac{1}{4} n^{2} r$, where $r=\Delta_{k}$.

Step $1(\mathbf{2} \leqslant \mathbf{i} \leqslant \mathbf{n}-\mathbf{1})$. Let $\omega_{i, k}=\varphi_{i, k}-\Sigma_{j=1}^{i-1} \frac{\partial \alpha_{i-1, k}}{\partial x_{j, k}} \varphi_{j, k}$. Because $\varphi_{1, k}, \cdots, \varphi_{i, k}$ are known smooth functions, there exist smooth function $\bar{\varphi}_{i, k}\left(x_{1, k}, \cdots, x_{i, k}, \hat{\eta}_{k}, \hat{S}_{k}\right)>0 \mathrm{such}$ that $\left|\delta^{\mathrm{T}}(t)\left(\varphi_{i, k}-\sum_{j=1}^{i-1} \frac{\partial \alpha_{i-1, k}}{\partial x_{j, k}} \varphi_{j, k}\right)\right| \leqslant\left\|\delta^{\mathrm{T}}(t)\right\| \bar{\varphi}_{i, k} \leqslant$ $s \bar{\varphi}_{i, k}$. Denote $z_{i+1, k}=x_{i+1, k}-\alpha_{i, k}-y_{r, k}^{(i)}, \tau_{i, k}=\tau_{i-1, k}+$ $\Gamma_{1} \phi(t) \omega_{i, k} z_{i, k}, \nu_{i, k}=\nu_{i-1, k}+\Gamma_{2} \frac{1}{\Delta_{k}} \bar{\varphi}_{i, k}^{2} z_{i, k}^{2}$. Then the time derivative of $z_{i, k}$ is given as

$$
\begin{gathered}
\dot{z}_{i, k}=z_{i+1, k}+\alpha_{i, k}+\eta^{\mathrm{T}} \phi(t) \omega_{i, k}+\delta^{\mathrm{T}}(t) \omega_{i, k}- \\
\frac{\partial \alpha_{i-1, k}}{\partial t}-\sum_{j=1}^{i-1} \frac{\partial \alpha_{i-1, k}}{\partial x_{j, k}} x_{j+1, k}- \\
\frac{\partial \alpha_{i-1, k}}{\partial \hat{\eta}_{k}} \dot{\hat{\eta}}_{k}-\frac{\partial \alpha_{i-1, k}}{\partial \hat{S}_{k}} \dot{\hat{S}}_{k} .
\end{gathered}
$$

Take virtual controller as

$$
\begin{aligned}
\alpha_{i, k}= & -z_{i-1, k}-c_{i} z_{i, k}-\hat{\eta}_{k}^{\mathrm{T}} \phi(t) \omega_{i, k}+\frac{\partial \alpha_{i-1, k}}{\partial t}+ \\
& \sum_{j=1}^{i-1} \frac{\partial \alpha_{i-1, k}}{\partial x_{j, k}} x_{j+1, k}+\frac{\partial \alpha_{i-1, k}}{\partial \hat{\eta}_{k}} \tau_{i, k}+\vartheta_{i, k}+ \\
& \frac{\partial \alpha_{i-1, k}}{\partial \hat{S}_{k}} \nu_{i, k}+\psi_{i, k}-\hat{S}_{k} \frac{1}{\Delta_{k}} \bar{\varphi}_{i, k}^{2} z_{i, k}
\end{aligned}
$$

where $\vartheta_{i, k}=\sum_{j=1}^{i-2} \frac{\partial \alpha_{j, k}}{\partial \hat{\eta}_{k}} \Gamma_{1} \phi(t) \omega_{i, k} z_{j+1, k}, \quad \psi_{i, k}=$ $\sum_{j=1}^{i-2} \frac{\partial \alpha_{j, k}}{\partial \hat{S}_{k}} \Gamma_{2} \frac{1}{\Delta_{k}} \bar{\varphi}_{i, k}^{2} z_{i, k} z_{j+1, k}$.

Choose the following nonnegative function:

$$
V_{i, k}\left(z_{k}, \hat{\eta}_{k}, \hat{S}_{k}\right)=\sum_{j=1}^{i} \frac{1}{2} z_{j, k}^{2}+\frac{1}{2} \tilde{\eta}_{k}^{\mathrm{T}} \Gamma_{1}^{-1} \tilde{\eta}_{k}+\frac{1}{2} \Gamma_{2}^{-1} \tilde{S}_{k}^{2} .
$$

The time derivative of $V_{i, k}$ along systems (11) is given, and (12) is substituted into it. We have

$$
\begin{aligned}
\dot{V}_{i, k} \leqslant & z_{i, k} z_{i+1, k}-\sum_{j=1}^{i} c_{j} z_{j, k}^{2}+ \\
& \left(\sum_{j=1}^{i-1} \frac{\partial \alpha_{j, k}}{\partial \hat{\eta}_{k}}\right) z_{i, k}\left(\tau_{i, k}-\dot{\hat{\eta}}_{k}\right)+ \\
& \left(\sum_{j=1}^{i-1} \frac{\partial \alpha_{j, k}}{\partial \hat{S}_{k}}\right) z_{i, k}\left(\nu_{i, k}-\dot{\hat{S}}_{k}\right)+ \\
& \tilde{\eta}_{k}^{\mathrm{T}} \Gamma_{1}^{-1}\left(\tau_{i, k}-\dot{\hat{\eta}}_{k}\right)+ \\
& \tilde{S}_{k} \Gamma_{2}^{-1}\left(\nu_{i, k}-\dot{\hat{S}}_{k}\right)+i \frac{1}{4} \Delta_{k} .
\end{aligned}
$$

Step $n$. Let $\omega_{n, k}=\varphi_{n, k}-\Sigma_{j=1}^{n-1} \frac{\partial \alpha_{n-1, k}}{\partial x_{j, k}} \varphi_{j, k}$. Because $\varphi_{1, k}, \cdots, \varphi_{n, k}$ are known smooth functions, there exist smooth function $\bar{\varphi}_{n, k}\left(x_{1, k}, \cdots, x_{n, k}, \hat{\eta}_{k}, \hat{S}_{k}\right)>0$ such that $\left|\delta^{\mathrm{T}}(t)\left(\varphi_{n, k}-\sum_{j=1}^{n-1} \frac{\partial \alpha_{n-1, k}}{\partial x_{j, k}} \varphi_{j, k}\right)\right| \leqslant\left\|\delta^{\mathrm{T}}(t)\right\| \bar{\varphi}_{n, k} \leqslant$ $s \bar{\varphi}_{n, k}$. Denote $\tau_{n, k}=\tau_{n-1, k}+\Gamma_{1} \phi(t) \omega_{n, k} z_{n, k}$ and $\nu_{n, k}=$ $\nu_{n-1, k}+\Gamma_{2} \frac{1}{\Delta_{k}} \bar{\varphi}_{n, k}^{2} z_{n, k}^{2}$. Then the time derivative of $z_{n, k}$ is given as

$$
\begin{aligned}
\dot{z}_{n, k}= & u_{k}-y_{r, k}^{(n)}+\eta^{\mathrm{T}} \phi(t) \omega_{n, k}\left(\bar{x}_{n, k}\right)+\delta^{\mathrm{T}}(t) \omega_{n, k}\left(\bar{x}_{n, k}\right)- \\
& \frac{\partial \alpha_{n-1, k}}{\partial t}-\sum_{j=1}^{n-1} \frac{\partial \alpha_{n-1, k}}{\partial x_{j, k}} x_{j+1, k}- \\
& \frac{\partial \alpha_{n-1, k}}{\partial \hat{\eta}_{k}} \dot{\hat{\eta}}_{k}-\frac{\partial \alpha_{n-1, k}}{\partial \hat{S}_{k}} \dot{\hat{S}}_{k}
\end{aligned}
$$

Take the following controller and adaptive iterative learning laws:

$$
\begin{aligned}
& u_{k}=\alpha_{n, k}+y_{r, k}^{(n)} \\
& \dot{\hat{\eta}}_{k}=\tau_{n, k} \\
& \dot{\hat{S}}_{k}=\nu_{n, k} .
\end{aligned}
$$

Choose the following nonnegative function:

$$
V_{n, k}\left(z_{k}, \hat{\eta}_{k}, \hat{S}_{k}\right)=\sum_{j=1}^{n} \frac{1}{2} z_{j, k}^{2}+\frac{1}{2} \tilde{\eta}_{k}^{\mathrm{T}} \Gamma_{1}^{-1} \tilde{\eta}_{k}+\frac{1}{2} \Gamma_{2}^{-1} \tilde{S}_{k}^{2} .
$$


The time derivative of $V_{n, k}$ along systems (15) is given, and after (16)-(18) are substituted into it, we have

$$
\dot{V}_{n, k} \leqslant-\sum_{j=1}^{n} c_{j} z_{j, k}^{2}+n \frac{1}{4} \Delta_{k}
$$

For the setting of the initial state, we give the following assumptions condition.

Assumption 1. For any $k$, when $t=0, x_{1, k}(0)=$ $y_{r, k}(0) ; x_{i+1, k}(0)=\alpha_{i, k}(0)-y_{r, k}^{(i)}(0), i=1, \cdots, n-1$; $\hat{\eta}_{k}(0)=\hat{\eta}_{k-1}(T) ; \hat{S}_{k}(0)=\hat{S}_{k-1}(T) ; y_{r, k}^{(i)}(0)=y_{r, k-1}^{(i)}(T)$, $i=1, \cdots, n$.

\subsection{Stability and convergence analysis}

Theorem 1. For nonlinear system (1) with assumption 1 , we design controller (16) and parameter adaptive laws (17) and (18). Then all signals of closed loop system are bounded on $[0, T]$, and we have

$$
\lim _{k \rightarrow \infty} z_{j, k}(t)=0, \quad j=1,2, \cdots, n .
$$

Proof. According to Assumption 1, we have $\left\|z_{k}(0)\right\|^{2}=$ $0 \leqslant\left\|z_{k}(T)\right\|^{2}$. By (19), we obtain

$$
\begin{aligned}
V_{n, k}\left(z_{k}(0), \hat{\eta}_{k}(T), \hat{S}_{k}(T)\right) \leqslant & V_{n, k}\left(z_{k}(0), \hat{\eta}_{k}(0), \hat{S}_{k}(0)\right)+ \\
& \int_{0}^{T} \dot{V}_{n, k} \mathrm{~d} t .
\end{aligned}
$$

Substitute (20) into (22), then

$$
\begin{aligned}
V_{n, k}\left(z_{k}(0), \hat{\eta}_{k}(T), \hat{S}_{k}(T)\right) \leqslant & V_{n, 1}\left(z_{1}(0), \hat{\eta}_{1}(0), \hat{S}_{1}(0)\right)- \\
& \sum_{i=1}^{k} \sum_{j=1}^{n} \int_{0}^{T} c_{j} z_{j, i}^{2} \mathrm{~d} t+ \\
& n\left(\frac{1}{4}\right) T\left(\sum_{i=1}^{k} \Delta_{i}\right) .
\end{aligned}
$$

Denote $V_{0}(k)=V_{n, 1}\left(z_{1}(0), \hat{\eta}_{1}(0), \hat{S}_{1}(0)\right)+n\left(\frac{1}{4}\right) T\left(\sum_{i=1}^{k} \Delta_{i}\right)$, then (23) can be rewritten as

$$
\sum_{i=1}^{k} \sum_{j=1}^{n} \int_{0}^{T} c_{j} z_{j, i}^{2} \mathrm{~d} t \leqslant V_{0}(k)-V_{n, k}\left(z_{k}(0), \hat{\eta}_{k}(T), \hat{S}_{k}(T)\right) .
$$

By (6), we have $\lim _{k \rightarrow \infty} V_{0}(k) \leqslant V_{n, 1}+2 a n\left(\frac{1}{4}\right) T$, then $V_{0}(k)$ is bounded, and $V_{n, k}\left(z_{k}(0), \hat{\eta}_{k}(T), \hat{S}_{k}(T)\right) \geqslant 0$, so

$$
\lim _{k \rightarrow \infty} \sum_{j=1}^{n} \int_{0}^{T} c_{j} z_{j, k}^{2} \mathrm{~d} t=0
$$

By (19), for any $k, V_{n, k}(t)=V_{n, k}(0)+\int_{0}^{t} \dot{V}_{n, k}(\tau) \mathrm{d} \tau$. Substitute (20) into the previous equations, then

$$
V_{n, k}(t) \leqslant V_{n, k}(0)-\sum_{j=1}^{n} \int_{0}^{t} c_{j} z_{j, k}^{2}(\tau) \mathrm{d} \tau+\operatorname{tn}\left(\frac{1}{4}\right) \Delta_{k} .
$$

By (25), $\sum_{j=1}^{n} \int_{0}^{t} c_{j} z_{j, k}^{2}(\tau) \mathrm{d} \tau$ is bounded. According to Definition $1, \Delta_{k}$ is bounded, and $t \in[0, T]$, so $\operatorname{tn}\left(\frac{1}{4}\right) \Delta_{k}$ is also bounded. And also $\hat{\eta}_{k}(0)=\hat{\eta}_{k-1}(T), \hat{S}_{k}(0)=\hat{S}_{k-1}(T)$.
By (23), for any $k, V_{n, k}\left(0, \hat{\eta}_{k}(T), \hat{S}_{k}(T)\right)$ is bounded, so $V_{n, k}\left(0, \hat{\eta}_{k}(0), \hat{S}_{k}(0)\right)=V_{n, k-1}\left(0, \hat{\eta}_{k-1}(T), \hat{S}_{k-1}(T)\right)$ is also bounded. From all above, for any $k, V_{n, k}(t)$ is bounded, then we have $x_{i, k}, \hat{\eta}_{k}(t)$ and $\hat{S}_{k}(t)$ are bounded. By (16), $u_{k}$ is bounded. By (11), $\dot{z}_{i, k}$ is bounded. So $z_{i, k}$ is continuously uniform, thus we get the conclusion of (21).

\section{An illustrative example}

In this section, an example is presented to show the effectiveness of the proposed adaptive iterative learning controller.

Consider the following second-order strict feedback nonlinear system:

$$
\begin{aligned}
& \dot{x}_{1, k}=x_{2, k}+\theta(t) x_{1, k}^{2} \\
& \dot{x}_{2, k}=u_{k}(t) \\
& y_{k}=x_{1, k}
\end{aligned}
$$

where $t \in[0,1], \theta(t)$ is systems uncertain periodical timevarying parameter. After it is expanded by Fourier series, system (27) can be described as

$$
\begin{aligned}
& \dot{x}_{1, k}=x_{2, k}+\eta^{\mathrm{T}} \phi(t) x_{1, k}^{2}+\delta^{\mathrm{T}}(t) x_{1, k}^{2} \\
& \dot{x}_{2, k}=u_{k}(t) \\
& y_{k}=x_{1, k}
\end{aligned}
$$

where $x_{1, k}$ and $x_{2, k}$ are state variables, $u_{k}(t)$ is the input variable. $\quad \eta=\left[\eta_{1}, \eta_{2}, \eta_{3}, \eta_{4}, \eta_{5}\right]^{\mathrm{T}}, \phi(t)=$ $[1, \sin (t), \cos (t), \sin (2 t), \cos (2 t)]^{\mathrm{T}},\|\delta(t)\| \leqslant s$, where $s$ is an unknown constant. In order to simulate, we suppose $\theta(t)=\sin (2 \pi t)$ in the actual system. Choose the reference trajectory $y_{r, k}=g_{k} \sin (2 \pi t)$ with difference amplitudes. For the non-uniform trajectory case, we choose $g_{k}=-0.2$ when $k$ is even, and $g_{k}=0.1$ when $k$ is odd.

Step 1. Let $z_{1, k}=x_{1, k}-y_{r, k}, z_{2, k}=x_{2, k}-\alpha_{1, k}-\dot{y}_{r, k}$, where the virtual control is taken as $\alpha_{1, k}=-c_{1} z_{1, k}-$ $\hat{\eta}_{k} \phi(t) x_{1, k}^{2}-\frac{1}{\Delta_{k}} \hat{S}_{k} x_{1, k}^{4} z_{1, k}^{2}, \Delta_{k}=\frac{a}{k^{2}}, \tau_{1, k}=\Gamma_{1} \phi(t) x_{1, k}^{2} z_{1, k}$, $\nu_{1, k}=\Gamma_{2} \frac{1}{\Delta_{k}} x_{1, k}^{4} z_{1, k}^{2}$.

Step 2. Design the controller $u_{k}(t)=-z_{1, k}-c_{2} z_{2, k}+$ $y_{r, k}^{(2)}-\hat{\eta}_{k}^{\mathrm{T}} \phi(t)\left|-\frac{\partial \alpha_{1, k}}{\partial x_{1, k}} x_{1, k}^{2}\right|+\frac{\partial \alpha_{1, k}}{\partial x_{1, k}} x_{2, k}+\frac{\partial \alpha_{1, k}}{\partial \hat{\eta}_{k}} \tau_{2, k}+$ $\frac{\partial \alpha_{1, k}}{\partial \hat{S}_{k}} \nu_{2, k}-\hat{\eta}_{k}^{\mathrm{T}} \dot{\phi}(t) x_{1, k}^{2}-\hat{S}_{k} \frac{1}{\Delta_{k}}\left|-\frac{\partial \alpha_{1, k}}{\partial x_{1, k}} x_{1, k}^{2}\right|^{2} z_{2, k}$, where $\Delta_{k}=\frac{a}{k^{2}}, \tau_{2, k}=\tau_{1, k}+\Gamma_{1} \phi(t)\left|-\frac{\partial \alpha_{1, k}}{\partial x_{1, k}} x_{1, k}^{2}\right| z_{2, k}, \nu_{2, k}=$ $\nu_{1, k}+\Gamma_{2} \frac{1}{\Delta_{k}}\left(\left|-\frac{\partial \alpha_{1, k}}{\partial x_{1, k}} x_{1, k}^{2}\right|\right)^{2} z_{2, k}^{2}$. Design the parameter adaptive laws: $\dot{\hat{\eta}}_{k}=\tau_{2, k}, \dot{\hat{S}}_{k}=\nu_{2, k}$.

Choose the following parameters and the initial values of states and the estimated parameters: $a=\frac{100}{3}, c_{1}=c_{2}=$ $0.1, \Gamma_{1}=\operatorname{diag}\{0.1\}, \Gamma_{2}=0.1, x_{1, k}(0)=0, x_{2, k}(0)=-0.4$, $\hat{\eta}_{k}(0)=[0,0,0,0,0]^{\mathrm{T}}, \hat{S}_{k}(0)=0$. For the iteration index $k=80$, the simulation results are shown in Figs. $1-5$.

Simulation results in Figs. $1-5$ show the effectiveness of the developed control scheme for system (27). From Figs. 1 and 2 , it can be seen that good tracking performance is obtained, that is to say, the tracking error can converge to zero. Moreover, The boundedness of the control signal $\left\|u_{k}\right\|$ is illustrated on the interval $[0,1]$ in Fig. 3. Parameter estimations $\left\|\hat{\eta}_{k}\right\|,\left\|\hat{S}_{k}\right\|$ are also bounded as given in Figs. 4 and 5 . 


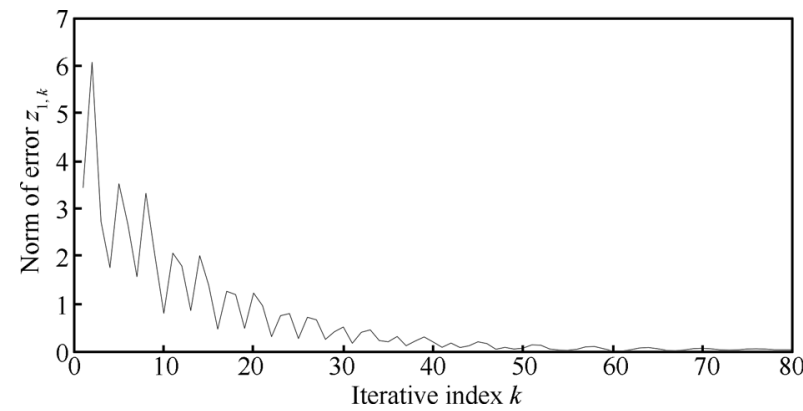

Fig. 1 Change of $\left\|z_{1, k}\right\|$ with iteration index

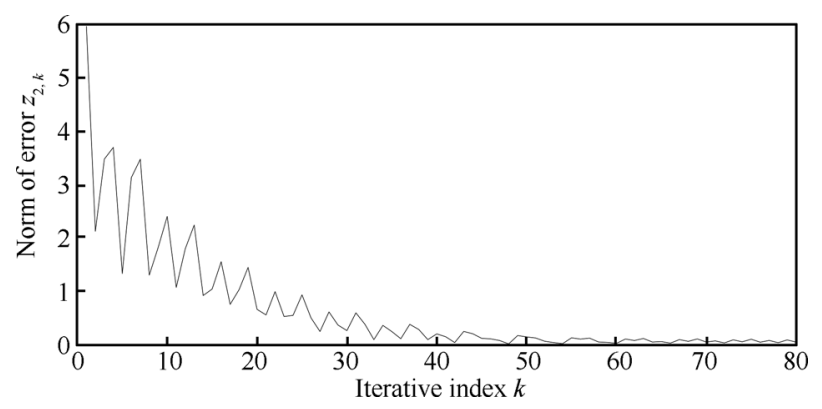

Fig. 2 Change of $\left\|z_{2, k}\right\|$ with iteration index

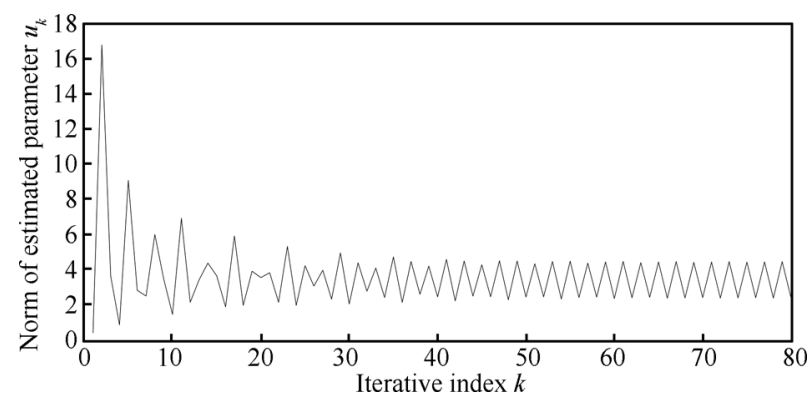

Fig. 3 Change of $\left\|u_{k}\right\|$ with iteration index

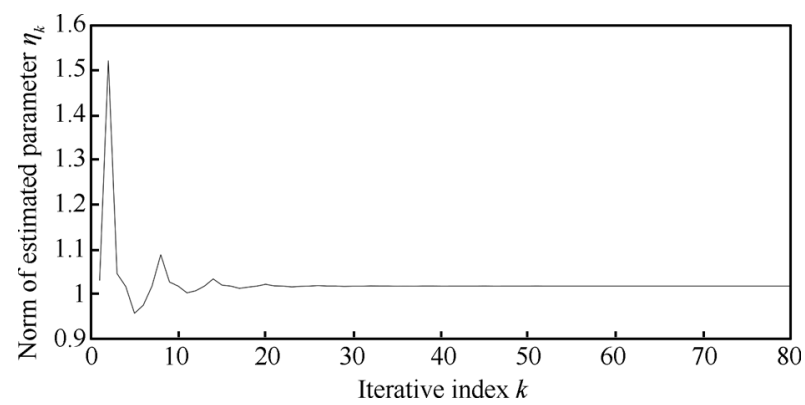

Fig. 4 Change of $\left\|\hat{\eta}_{k}\right\|$ with iteration index

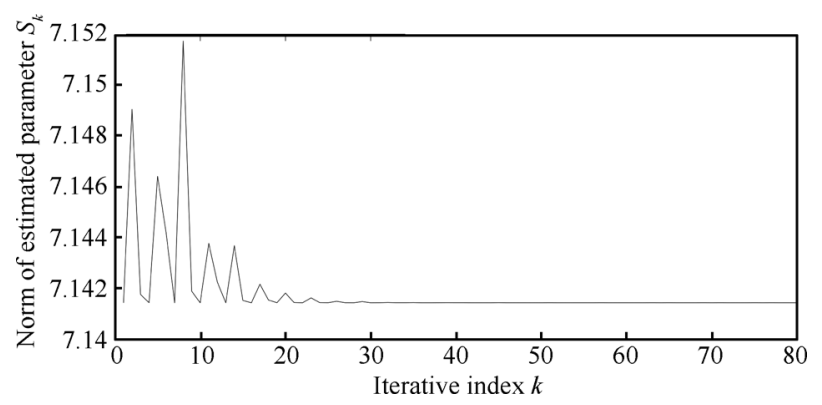

Fig. 5 Change of $\left\|\hat{S}_{k}\right\|$ with iteration index

\section{Conclusions}

This paper deals with the non-uniform trajectory tracking problem of a class of high-order nonlinear systems with unknown time-varying parameters. An adaptive iterative learning controller is designed for nonlinear systems with unknown time-varying parameters to realize the non-uniform trajectory tracking perfectly. Then, based on the Lyapunov stability theory, the asymptotic tracking of the controlled nonlinear system is proved. Simulation results demonstrate the effectiveness of the proposed control method. The future work is how to design the iterative learning controller for nonlinear systems with unknown time-varying parameters and uncertain control direction.

\section{References}

[1] H. S. Ahn, C. H. Choi, K. B. Kim. Iterative learning control for a class of nonlinear systems. Automatica, vol. 29, no. 6, pp. 1575-1578, 1993.

[2] C. J. Chien, J. S. Liu. A P-type iterative learning controller for robust output tracking of nonlinear time-varying systems. International Journal of Control, vol. 64, no. 2, pp. 319-334, 1996.

[3] W. G. V. Samer, S. Saab, M. H. Mickle. Learning control algorithms for tracking slowly varying trajectories. IEEE Transactions on Systems, Man, Cybernetics B, vol. 27, pp. 657-670, 1997.

[4] M. French, E. Rogers. Non-linear iterative learning by an adaptive Lyapunov technique. International Journal of Control, vol. 73, no. 10, pp. 840-850, 2000.

[5] X. J. Yang, J. M. Li. Intelligent iterative learning control for a class of unknown nonlinear systems. Control and Decision, vol. 16, no. 3, pp. 360-363, 2002. (in Chinese)

[6] J. X. Xu, J. Xu. On iterative learning from different tracking tasks in the presence of time-varying uncertainties. IEEE Transactions on Systems, Man, Cybernetics, Part B, vol. 34 , no. 1, pp. 589-597, 2004.

[7] J. M. Li, Y. P. Sun, Y. Liu. Hybrid adaptive iterative learning control of non-uniform trajectory tracking. Control Theory and Applications, vol. 25, no. 1, pp. 100-104, 2008.

[8] J. M. Li, X. M. Li, K. Y. Xing. Hybrid adaptive iterative learning control of non-uniform trajectory tracking for nonlinear time-delay systems. In Proceedings of the 26th Chinese Control Conference, IEEE, Zhangjiajie, China, pp. 515-519, 2007.

[9] C. L. Zhang, J. M. Li. Adaptive iterative learning control for nonlinear time-delay systems with periodic disturbances using FSE-neural network. International Journal of Automation and Computing, vol. 8, no. 4, pp. 403-410, 2011.

[10] D. Li, J. M. Li. Adaptive iterative learning control for nonlinearly parameterized systems with unknown time-varying delay and unknown control direction. International Journal of Automation and Computing, vol.9, no. 6, pp.578-586, 2012 . 
[11] H. Y. Yue, J. M. Li. Adaptive fuzzy dynamic surface control for a class of perturbed nonlinear time-varying delay systems with unknown dead-zone. International Journal of $\mathrm{Au-}$ tomation and Computing, vol. 9, no. 5, pp. 545-554, 2012.

[12] J. X. Xu. A new periodic adaptive control approach for time-varying parameters with known periodicity. IEEE Transactions on Automatic Control, vol. 49, no. 4, pp. 579$583,2004$.

[13] R. Yan, M. J. Er, Y. J. Pan. Multi-period repetitive learning control for a class of unmatched systems with unknown control direction. In Proceedings of American Control Conference, IEEE, Minneapolis, MN, USA, pp. 238-243, 2006.

[14] J. Zhao, X. F. Wu, D. F. Tang, Z. Q. Wang, Y. P. Sun. Adaptive backstepping learning control for a class of nonlinear time-varying systems. In Proceedings of International Workshop on Intelligent Systems and Applications, IEEE, Wuhan, China, pp. 1-5, 2009.

[15] S. Zhu, M. X. Sun, X. X. He. Iterative learning control of strict-feedback nonlinear time-varying systems. Acta Automatica Sinica, vol. 36, no. 3, pp. 454-458, 2010.

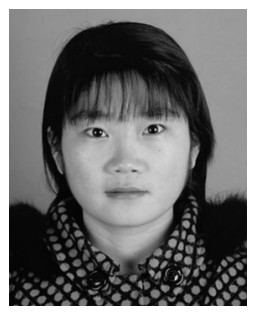

responding author)

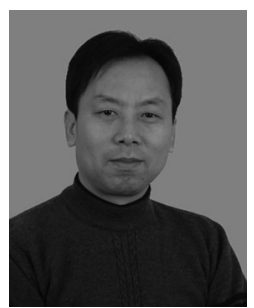

Jun-Min Li graduated from Xidian University, China in 1987 . He received his M. Sc. degree from Xidian University in 1990 and his $\mathrm{Ph} . \mathrm{D}$. degree from the $\mathrm{Xi}^{\prime}$ an Jiaotong University, China in 1997, respectively. He is currently a professor at Department of Applied Mathematics, Xidian University

His research interests include adaptive control, learning control, intelligent control, hybrid system control theory, and the networked control systems. E-mail: jmli@mail.xidian.edu.cn
Chun-Li Zhang graduated from Linyi Normal University, China in 2007. She reversity, China in 2010. She is currently a Ph. D. candidate at Department of Applied

Her research interests include adaptive iterative learning control, neural network, bust control and chaos synchronization.

E-mail: gaozhangchunli@163.com (Cor- 